Supporting Information

\title{
Superamphiphobic Silicon Nanowires Embedded Microsystem and In-Contact Flow Performance of Gas and Liquid Streams
}

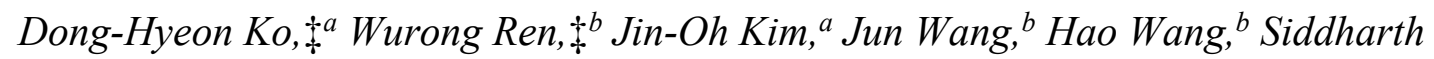
Sharma, ${ }^{a}$ Marco Faustini, ${ }^{c}$ and Dong-Pyo Kim ${ }^{a *}$

${ }^{a}$ National Center of Applied Microfluidic Chemistry, Department of Chemical Engineering, Pohang University of Science and Technology (POSTECH), Environ. Eng. Bldg., 77, Cheongam-ro, Nam-gu, Pohang-si, Gyungsangbuk-do, 37673, South Korea

${ }^{b}$ Science and Technology on Advanced Ceramic Fibers and Composites Laboratory, College of Aerospace Science and Engineering, National University of Defense Technology, Changsha 410073, Hunan Province, PR China

${ }^{c}$ Sorbonne Universités, UPMC Univ Paris 06, CNRS, CNRS, Collège de France, UMR 7574, Chimie de la Matière Condensée de Paris, F-75005, Paris, France

* E-mail: dpkim@postech.ac.kr 


\section{Materials}

Si wafer (P/100, dopant: boron) was purchased from Semi-Materials Co., Ltd.; Hydrofluoric acid (HF) solution (48-51 wt. \%) was purchased from Avantor Performance Materials, Inc; Hydrogen peroxide $\left(\mathrm{H}_{2} \mathrm{O}_{2}\right)$ solution 30 wt. \%, ammonia solution 28-30 wt. \% were purchased from Samchun Pure Chemical Co., Ltd; Dimethyl sulfoxide (DMSO) $99.5 \%$ was purchased from Daejung Chemicals and Metals Co., Ltd; AZ 1512 positive photoresist and the developer was purchased from AZ Electronic Materials; SU-8 50 negative photoresist was purchased from K1 Solutions; Polydimethylsiloxane (PDMS) (Sylgard 184) was purchased from Dow Corning; Allylhydridopolycarbosilane (AHPCS) was purchased from Starfire Systems; Silver nitrate $99 \%$, Trichloro $(1 \mathrm{H}, 1 \mathrm{H}, 2 \mathrm{H}, 2 \mathrm{H}$-perfluorooctyl)silane (FOTS) $97 \%$, Palladium (II) acetate $98 \%$, Phenylboronic acid $97 \%$, Ethyl acrylate $99 \%$, Benzaldehyde $99.5 \%$, 2-Aminolthiophenol $99 \%$, Eosin-Y dye as a photocatalyst, Tetraethyl orthosilicate $99 \%$ (TEOS), Hydrochloric acid $37 \%$, Copper sulfate, Phenylboronic acid, Palladium acetate, [2,2']-bipyridine, N,N-dimethylformamide (DMF), N-ethylbutylamine $98 \%$, Acetonitrile anhydrous $99.8 \%$ were purchased from Sigma Aldrich. The deionized water (18.2 mS conductivity) was used for every experiments. 


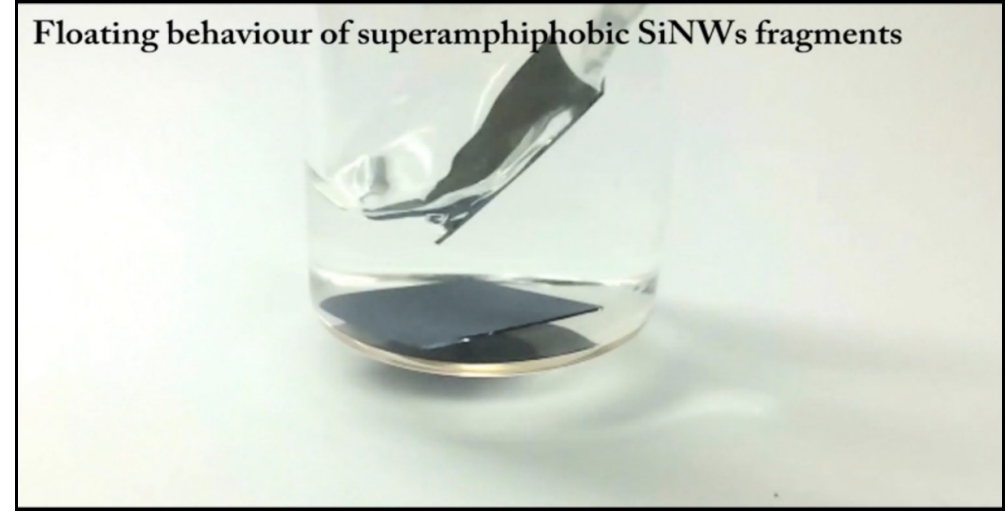

Supporting Movie 1. Flotation behavior of SiNWs fragment. When dropped onto liquids (water, DMSO, hexadecane) and slightly immersed into the liquids at the begining, then easily floated on the surface of liquids. In contrary, the bare Si wafer was immediately immersed into the liquids. When the liquids were poured into empty beaker containging both the SiNWs and the bare Si fragments, only SiNWs fragment moved up to float on the surface. Upon thoroughly shaking, SiNWs fragment didn’t sink down. 


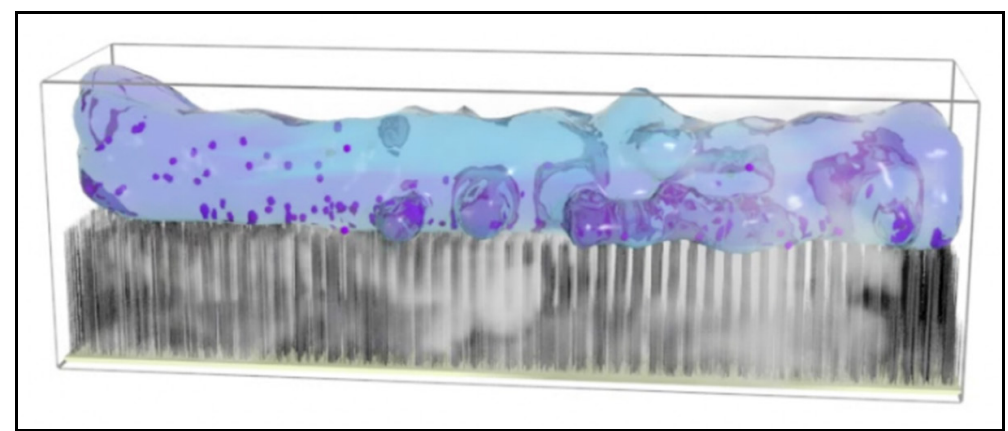

Supporting Movie 2. Animation on a flow principle of gas-lqiuidc binary phase a SiNWs-equipped membrane-free microreactor (SEMFM), in which both gas and liquid phase maintain stable interface to form laminar flow due to superamphiphobicity of the SiNWs structures. 


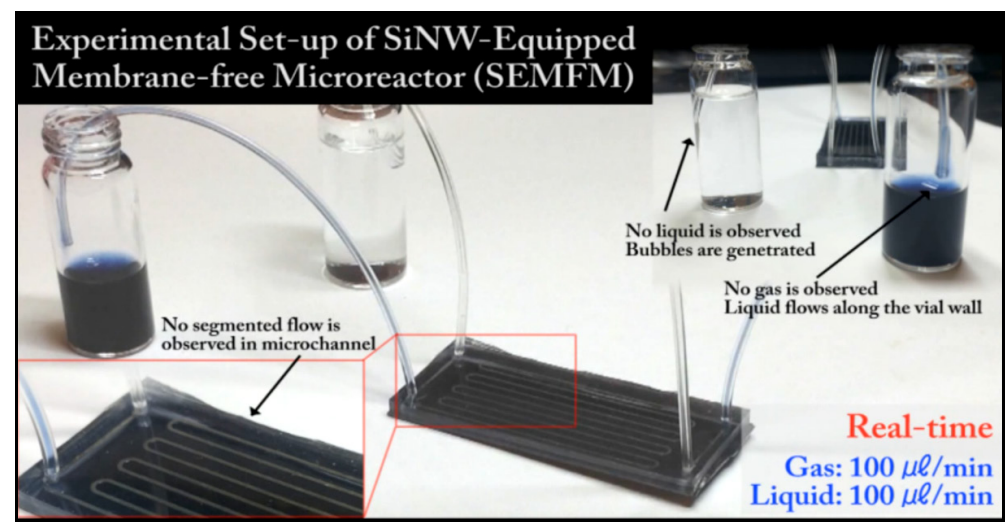

Supporting Movie 3. Experimental movie with 3 views (standard, vial-close, and magnified view) of a superamphiphobic SiNWs-equipped membrane-free microreactor. The gas-liquid laminar flow was generated by injecting both gas and liquid at flow rate of $100 \mathrm{and} 100 \mu \mathrm{L} / \mathrm{min}$, respectively.Liquid flow with no bubble was observed atbothmicrochannel and liquid outlet, and rose the liquid level in vial by slowly flowing down along the vial wall. Gas bubble was also observed only at gas outlet that was immersed into a vial of pure water. It confirmsthat either gas bubble or dyed solution did not cross over the interface to maintain the stable laminar flow although there was no physical barrier. 


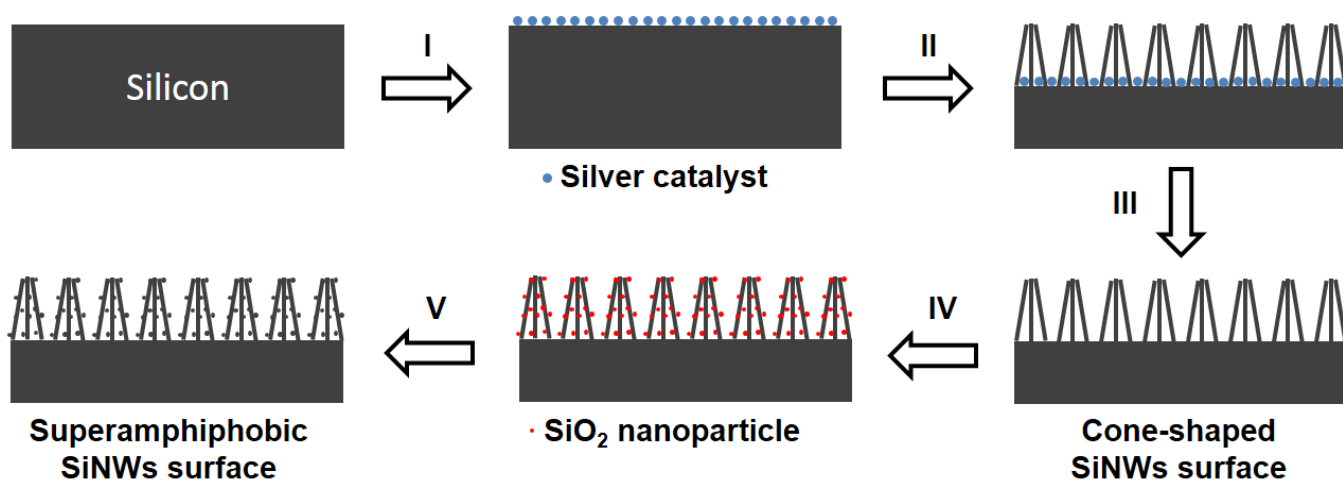

Figure S1. Schematic illustration of fabrication process of cone-shaped superamphiphobic Si nanowires (SiNWs) surface, I: loading silver catalyst on Si surface, II: silver aided chemical etching to form a cone-shaped SiNW pattern, III: silver catalyst removal, IV: decoration of $\mathrm{SiO} 2$ nanoparticles on SiNW surface to form nano-roughened surface, V: fluorination of SiNW surface. 

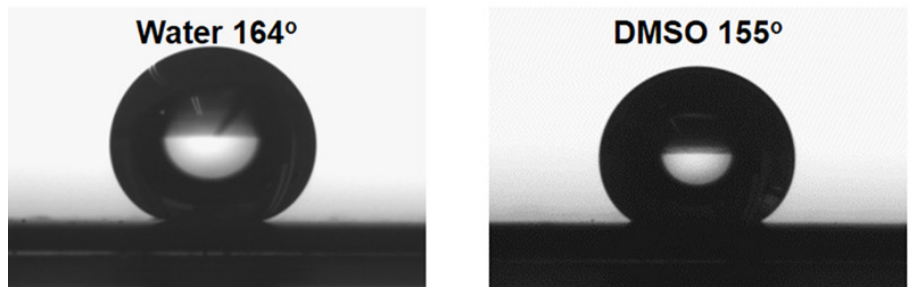

\section{Hexadecane $120^{\circ}$}

Figure S2. Static contact angle of water, DMSO and hexadecane on superamphiphobic SiNW surfaces. Liquid droplets of ca. $5 \mu \mathrm{L}$ were used and Young-Laplace curves were employed for fitting process determining the contact angles. 
a)
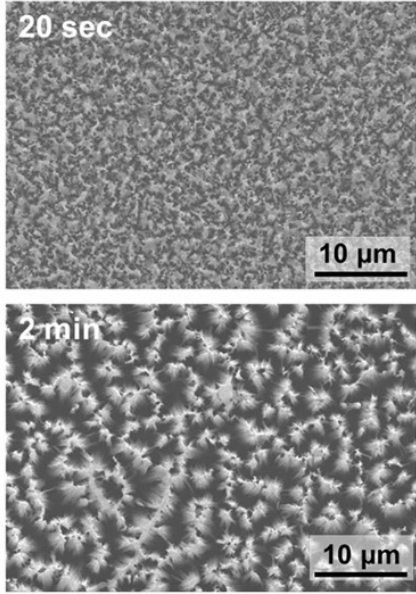

b)
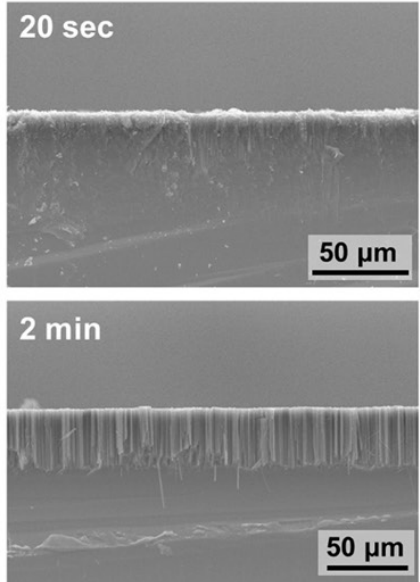

$50 \mu \mathrm{m}$

c)

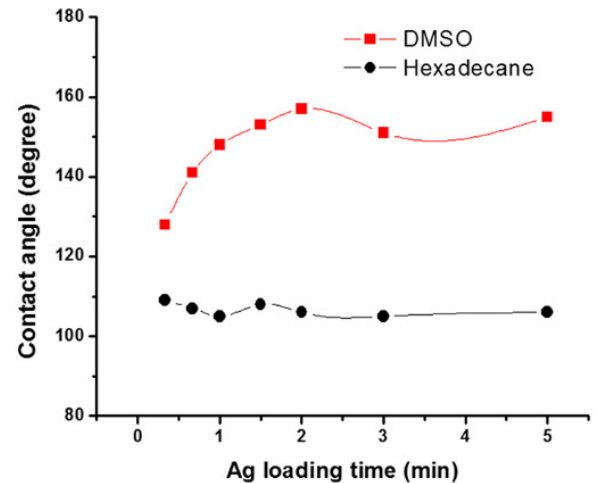

Ag loading time (min)

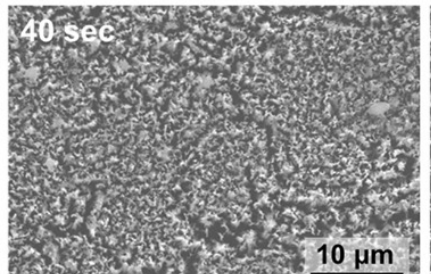

$10 \mu \mathrm{m}$
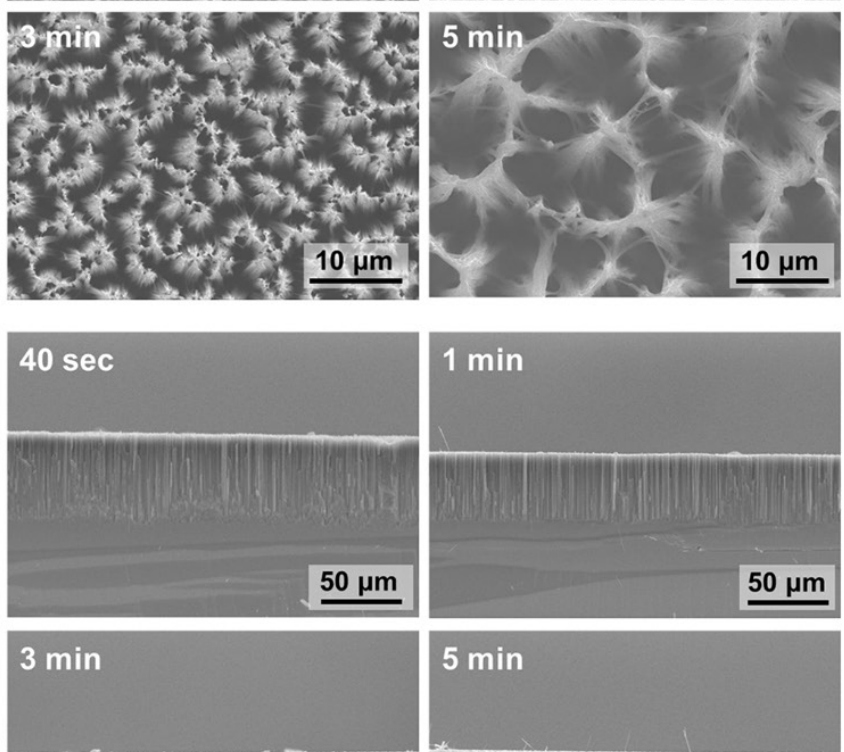

$5 \mathrm{~min}$

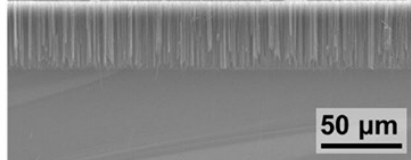

$50 \mu \mathrm{m}$

$50 \mu \mathrm{m}$

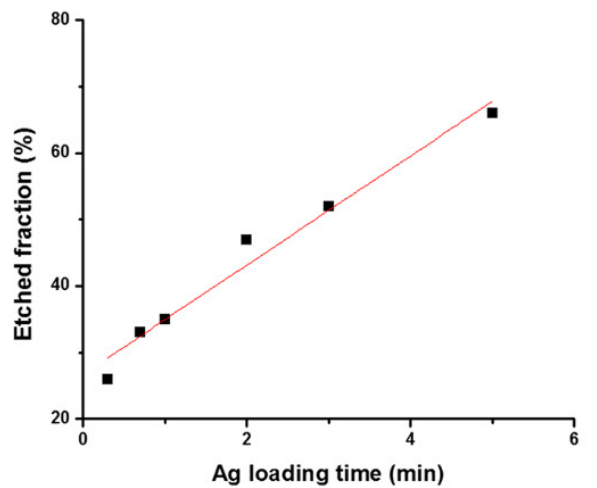

d)

\begin{tabular}{|c|c|c|c|c|c|c|c|}
\hline Silver loading time (sec) & 20 & 40 & 60 & 90 & 120 & 180 & 300 \\
\hline $\begin{array}{c}\text { Optical image } \\
\text { (scale bar: } 500 \mu \mathrm{m})\end{array}$ & $\ldots$ & - & - & - & - & - & \\
\hline
\end{tabular}

Figure S3. Various SiNWs surface morphology and wetting behavior by varying silver catalyst loading time (20 sec 5 min) when $\mathrm{HF}$ etching time and TEOS concentration for $\mathrm{SiO}_{2}$ decoration were fixed at $4 \mathrm{hr}$ and $24 \mathrm{mM}$, respectively. All CA measurements were conducted after fluorination. Vertically aligned SiNWs morphology was maintained upon less than 2 min of $\mathrm{t}_{\mathrm{Ag}}$ with etched fraction ca. $42 \%$. When $\mathrm{t}_{\mathrm{Ag}}$ was extended to $5 \mathrm{~min}$, etched fraction $66 \%$ significantly lowered the SiNW density with high aspect ratios to be aggregated presumably due to capillary force, forming a cone-shaped SiNWs morphology that is required for the superoleophobic surface property. In case over 5 min of tAg, the SiNWs partly started detaching from the substrate. a) SEM images of top view and b) cross-section view. c) Contact angles of DMSO and hexadecane and etched fraction of Si. d) Optical images of SiNWs samples with various silver loading time. 
a)

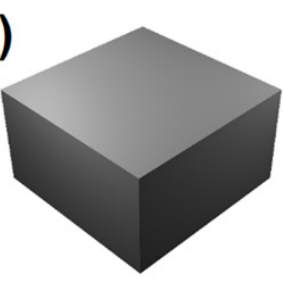

b)
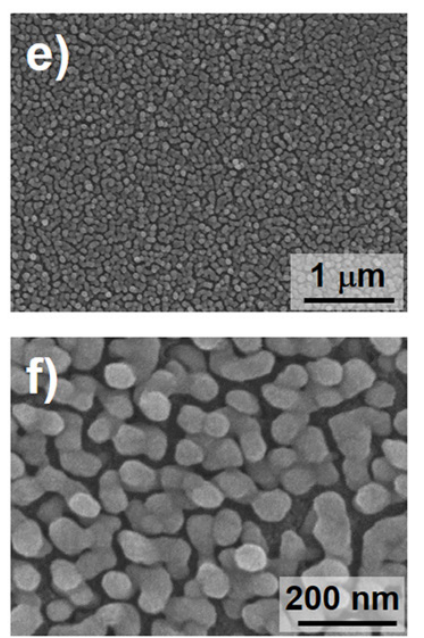

c)

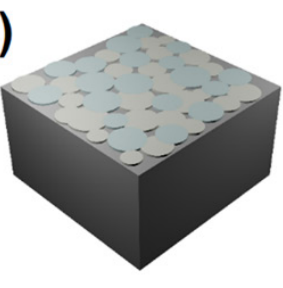

d)

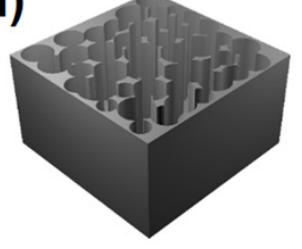

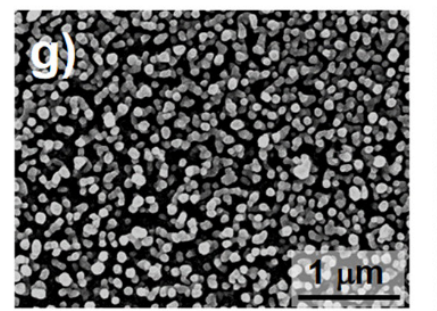
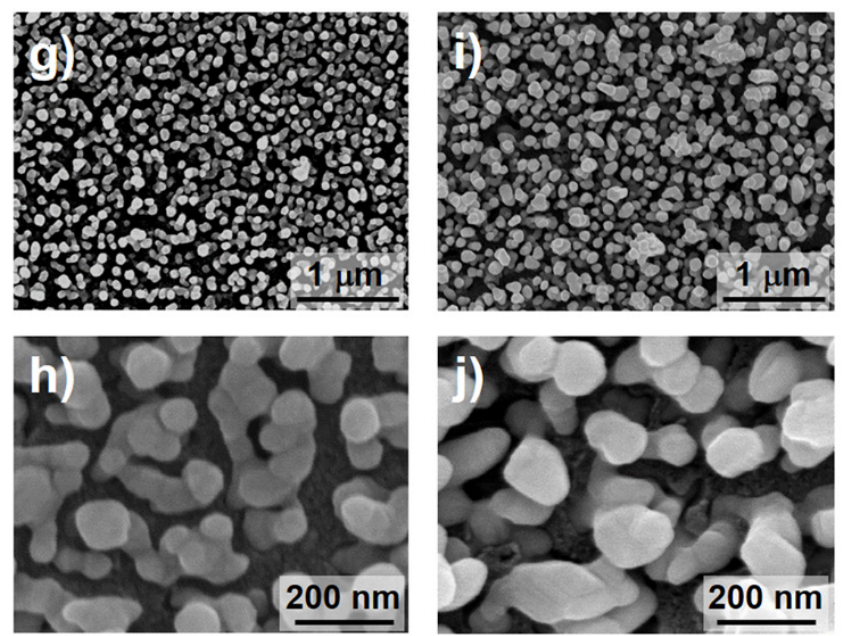

Figure S4. a)-d) Schematic illustrations for denser silver catalyst deposit by longer loading time, then caused sparser SiNWs after etching. In general, longer loading of silver catalyst caused denser deposition of silver, which leaded to sparser formation of SiNWs by higher etched fraction. e)-j) SEM images of silver catalyst deposited at different loading times. 1 min for e)\& f), 3 min for g) \& h), and 5 min for i)\& j). 
a)
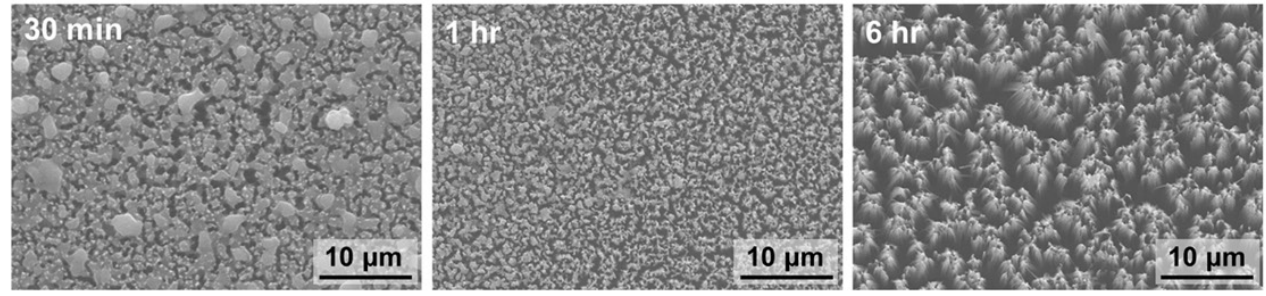

b)
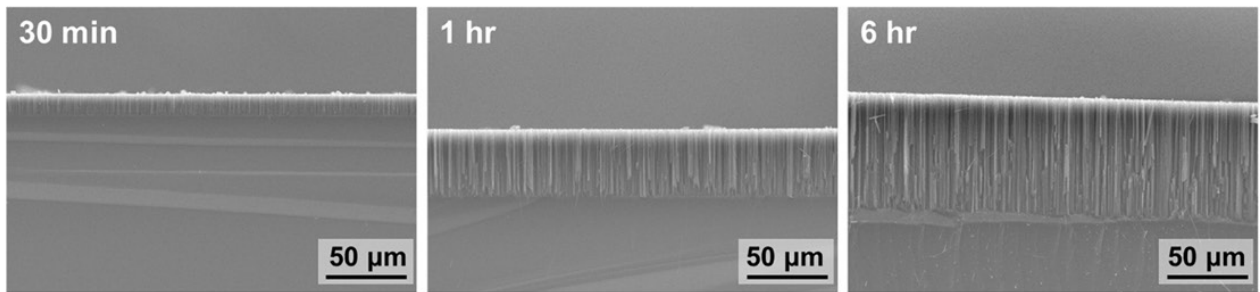

c)

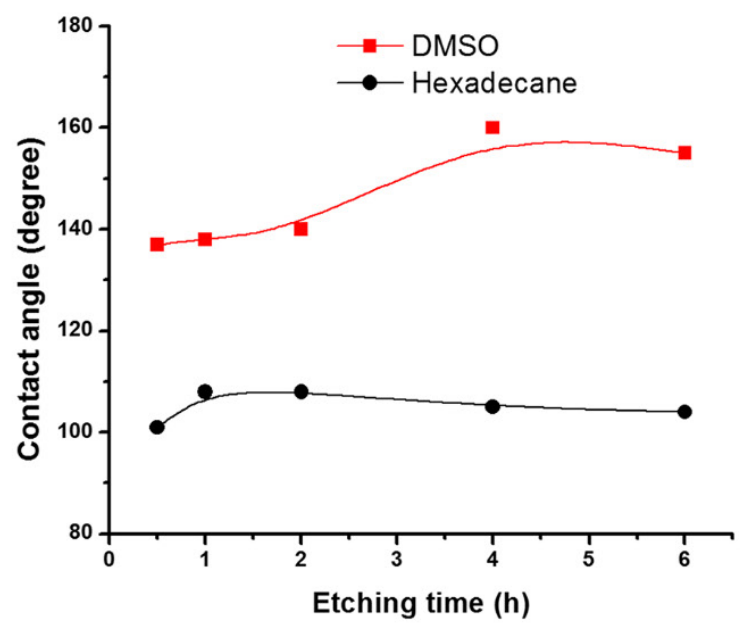

d)
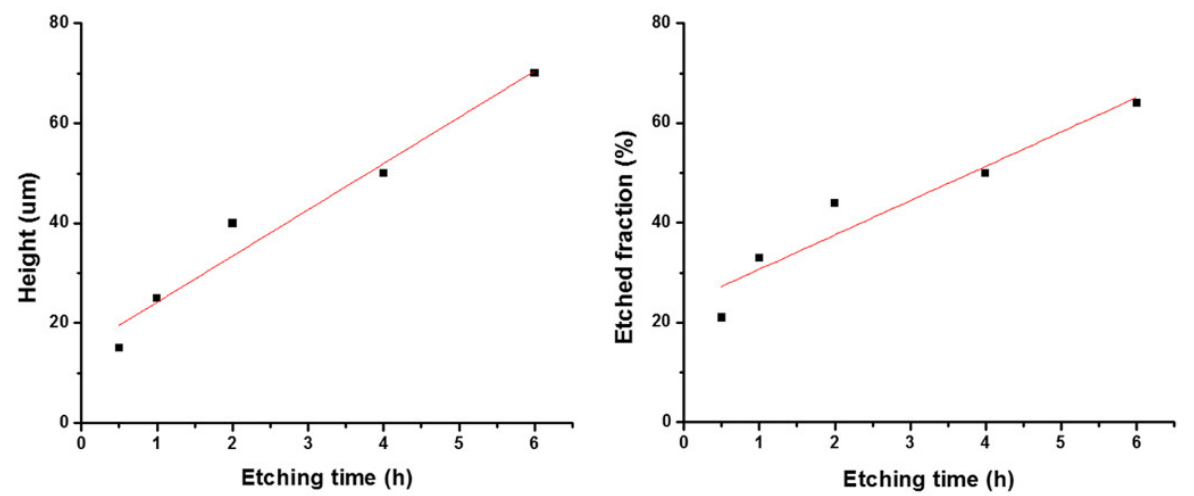

Figure S5. Optimization of superamphiphobic SiNWs surface by varying chemical etching time from 0.5 to $5 \mathrm{hr}$ when silver loading time and TEOS concentration (for $\mathrm{SiO}_{2}$ decoration) were fixed at $2 \mathrm{~min}$ and $24 \mathrm{mM}$, respectively. a) SEM images of top view and b) crosssectional view. c) CAs of DMSO and hexadecane after fluorination, d) heights and etched fraction of chemically etched SiNWs. 
a)
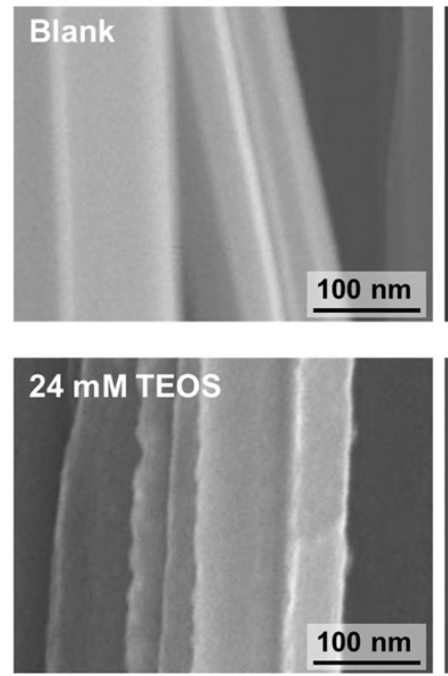
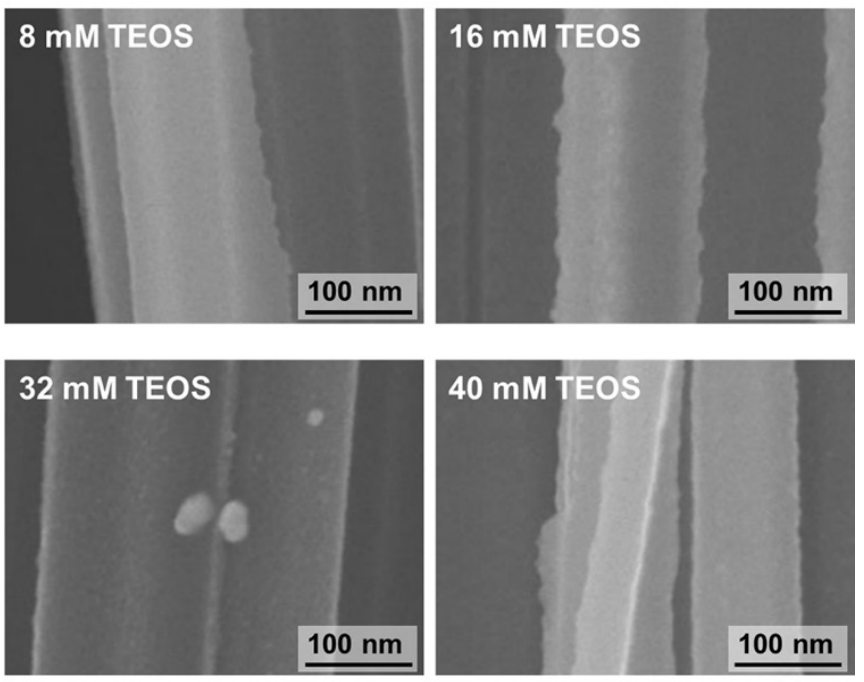

b)

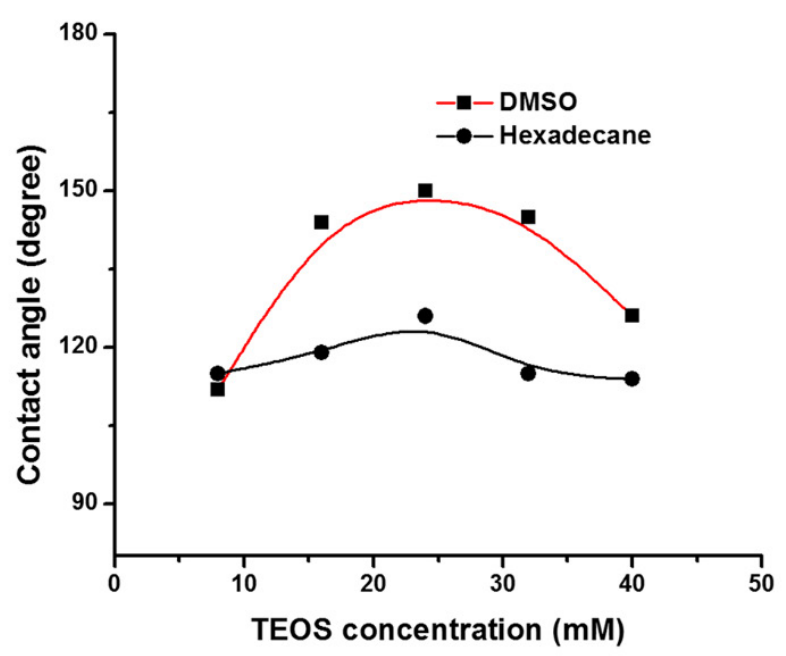

Figure S6. Optimization of fluorinated superamphiphobic SiNWs surfaceby varying TEOS concentration for $\mathrm{SiO}_{2}$ decoration from 8 to $40 \mathrm{mM}$ when silver loading time and chemical etching time were fixed at $2 \mathrm{~min}$ and $4 \mathrm{hr}$, respectively a) SEM images. b) CAs of DMSO and hexadecane after fluorination. 

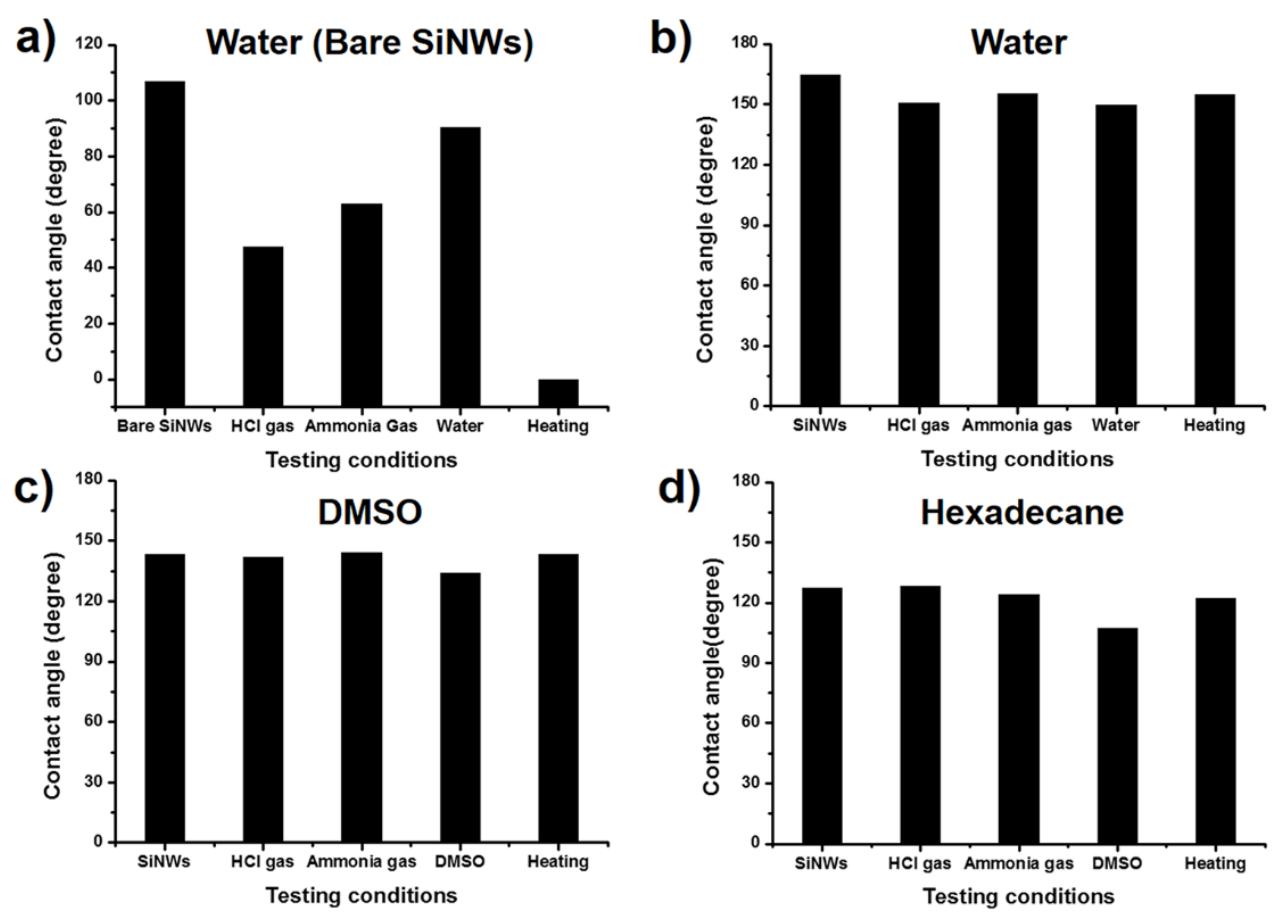

Figure S7. Chemical and thermal stability of superamphiphobic SiNWs after exposure to various stress conditions, measured the contact angle before and after the stress. a) Water CA of SiNWs without surface fluorination treatment. b) Water CA, c) DMSO CA, and d) hexadecane CA on the fluorinated SiNWs. 
a)

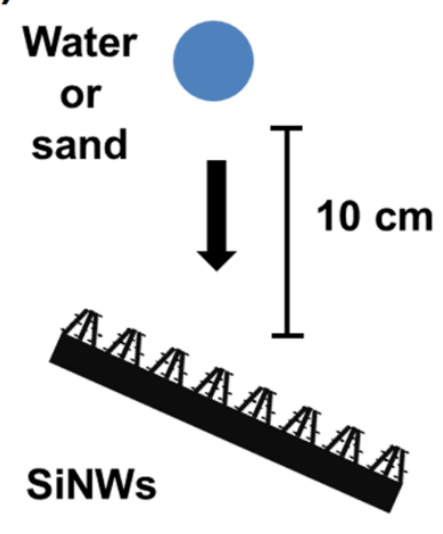

b)

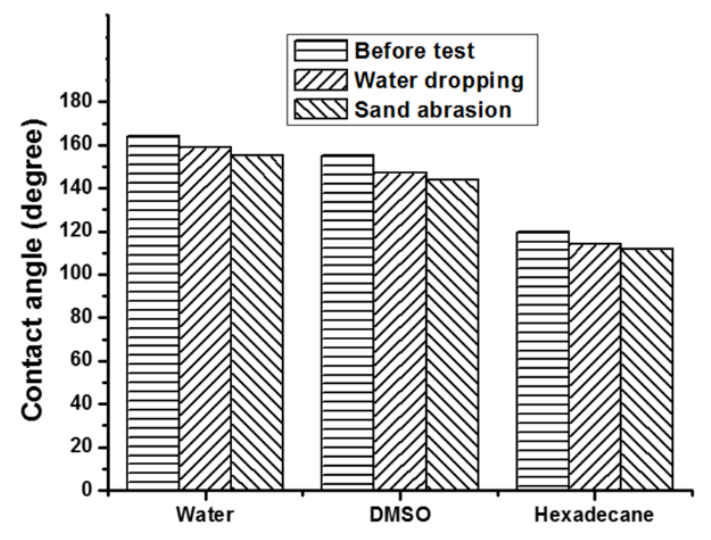

Figure S8. Mechanical stability of superamphiphobic SiNWs after water dropping and sand abrasion experiments. a) Schematic diagram of the test conditionsfor water (60 psi for $1 \mathrm{hr}$ ) and sand (grain diameter 100 300 $\mu \mathrm{m}$, amount $20 \mathrm{~g}$ in $10 \mathrm{sec}$ ). b) CA of superamphiphobic SiNWs after mechanical stability tests. 

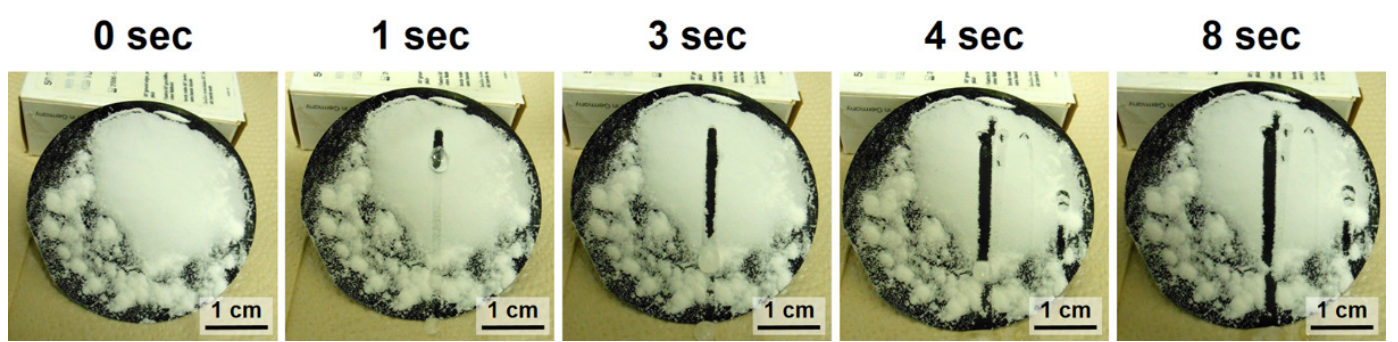

Figure S9. Self-cleaning property of 3"wafer-scale superamphiphobic SiNWs, when a glycerol liquid was dropped on AlOOH stained SiNWs surface, white AlOOH particles was completely removed. 
a)
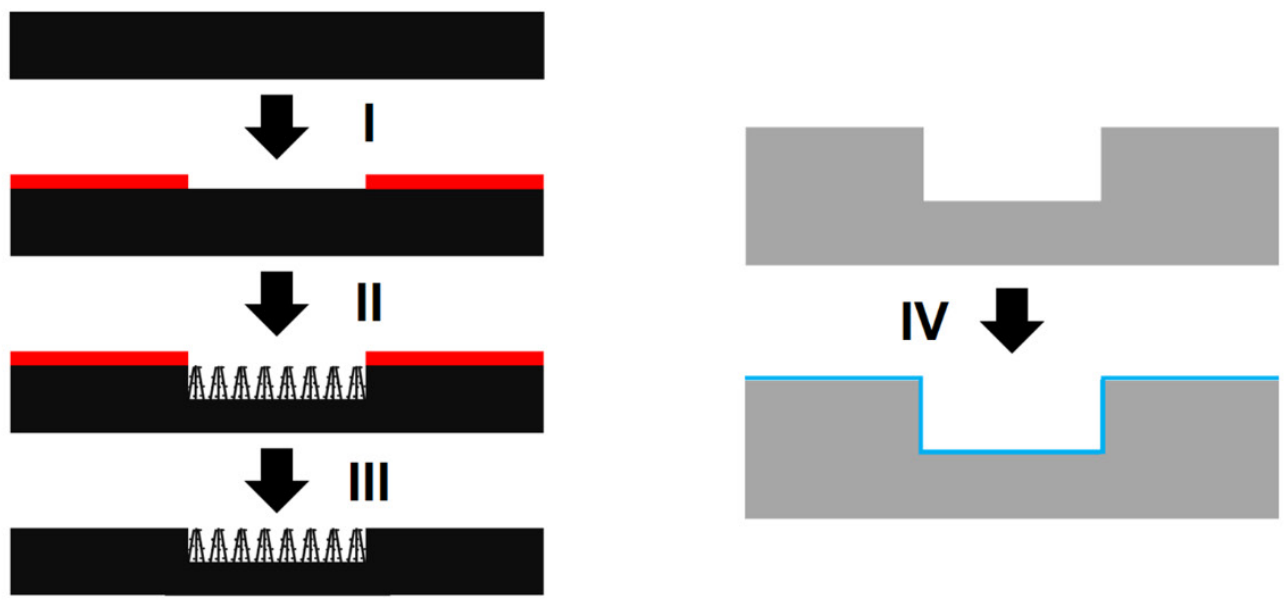

$\checkmark v$

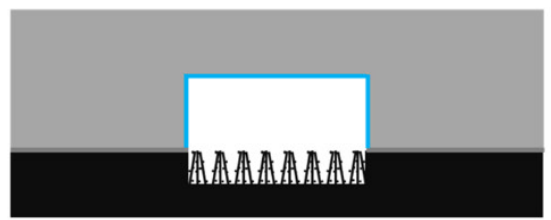

b)
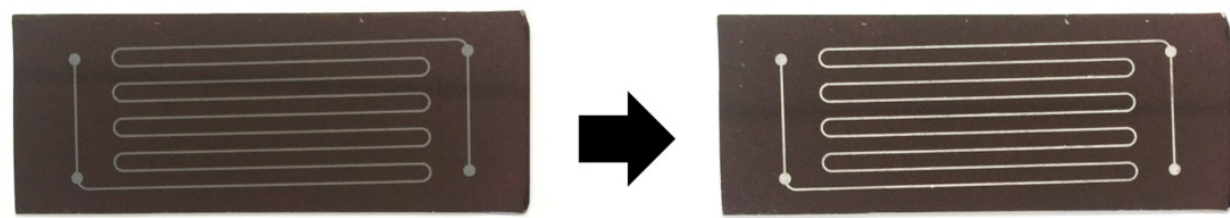

$1 \mathrm{~cm}$

AZ patterning on $\mathrm{Si}$

Ag loading on unprotected Si
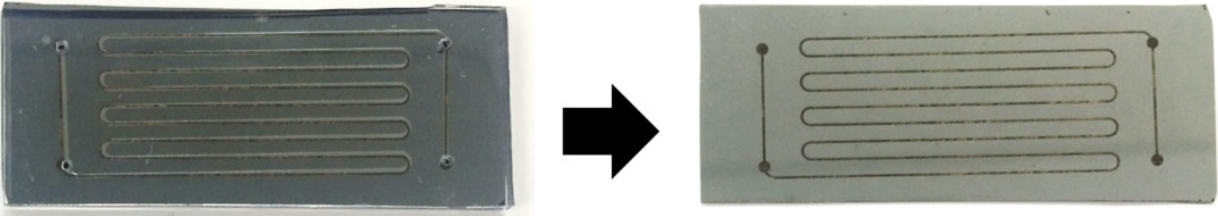

$1 \mathrm{~cm}$

SiNW membrane-free microreactor

\section{Serpentine SiNWs pattern}

Figure S10. a) Schematic fabrication process of superamphiphobic SiNWs-equipped membrane-free microreactor. I: patterning of AZ photoresist protective layer on $\mathrm{Si}$, II: preparation of superamphiphobic SiNWs pattern, III: cleaning AZ photoresist layer, IV: spin-coating allylhydridopolycarbosilane (AHPCS) adhesive ( $3 \mu \mathrm{m}$ thick) at $3000 \mathrm{rpm}$ for $30 \mathrm{sec}$ on plasma treated PDMS channel, V: adhesive bonding of AHPCS coated PDMS channel with superamphiphobic SiNWs pattern by UV exposure for $10 \mathrm{~min}$ and heating at $150{ }^{\circ} \mathrm{C}$ for $3 \mathrm{hr} . \mathrm{b}$ ) Optical images of AZ pattern and Ag loading on Si (after Step I), superamphiphobic serpentine SiNWs pattern (after Step III) and SiNW membrane-free microreactor (after Step V). 


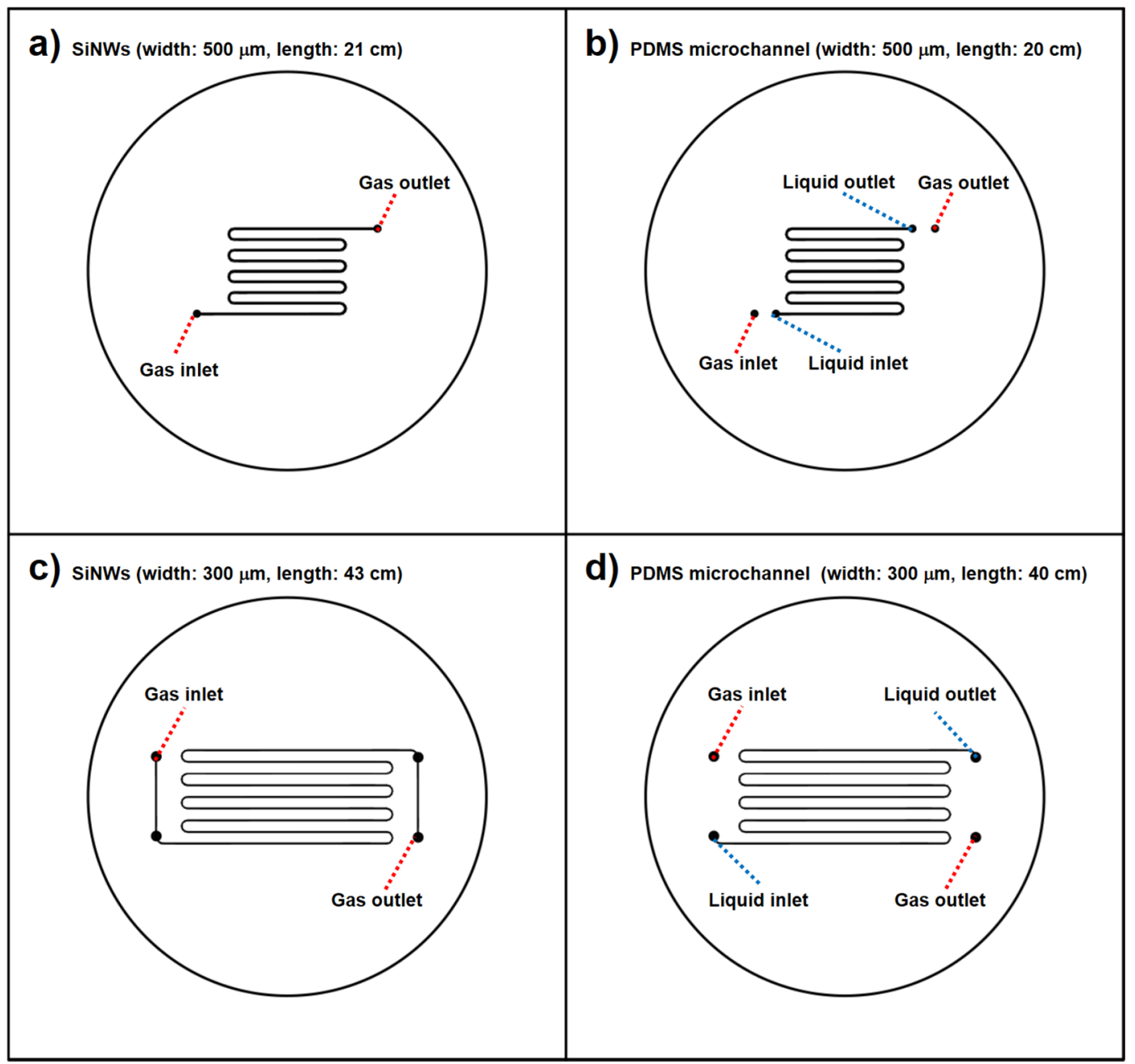

Figure S11. Photomask patterns for serpentine SiNWs and PDMS microchannel. $500 \mu \mathrm{m}$ wide and $20 \mathrm{~cm}$ long patterns for a) SiNWs and b) PDMS microchannel. $300 \mu \mathrm{m}$ wide and $40 \mathrm{~cm}$ long patterns for c) SiNWs and d) PDMS microchannel. Photomask for PDMS patterns lack the line between gas inlet (or outlet) and liquid inlet (or outlet) to separate routes for gas and liquid phases. 